\title{
Proxemics with Multiple Dynamic Characters in an Immersive Virtual Environment
}

\author{
JOAN LLOBERA \\ Starlab Barcelona -Universitat de Barcelona \\ BERNHARD SPANLANG \\ Universitat Politècnica de Catalunya \\ GIULIO RUFFINI \\ Starlab Barcelona \\ and \\ MEL SLATER \\ ICREA - Universitat de Barcelona - Universitat Politècnica de Catalunya -
}

Categories and Subject Descriptors: I.3.7 [Computer Graphics]: Three-Dimensional Graphics and RealismVirtual reality.

\begin{abstract}
An experiment was carried out to examine the impact on electrodermal activity of people when approached by groups of 1 or 4 virtual characters to varying distances. It was premised on the basis of proxemics theory that the closer the approach of the virtual characters to the participant, the greater the level of physiological arousal. Physiological arousal was measured by the number of skin conductance responses within a short time period after the approach, and the maximum change in skin conductance level $5 \mathrm{~s}$ after the approach. The virtual characters were each either female or a cylinder of human size, and 1 or 4 characters approached each subject a total of 12 times. Twelve male subjects were recruited for the experiment. The results suggest that the number of skin conductance responses after the approach and the change in skin conductance level increased the closer the virtual characters approached towards the participants. Moreover, these response variables were inversely correlated with the number of visits, showing a typical adaptation effect. There was some evidence to suggest that the number of characters who simultaneously approached (1 or 4) was positively associated with the responses. Surprisingly there was no evidence of a difference in response between the humanoid characters and cylinders on the basis of these physiological data. It is suggested the similarity in this quantitative arousal response to virtual characters and virtual objects might mask a profound difference in qualitative response, an interpretation supported by questionnaire and interview results. Overall the experiment supported the premise that people exhibit heightened physiological arousal the closer they are approached by virtual characters.
\end{abstract}

Categories and Subject Descriptors: I.3.7 [Computer Graphics]: Three-Dimensional Graphics and RealismVirtual reality

General Terms: Experimentation, Human Factors

Additional Key Words and Phrases: human-computer interaction, proxemics, virtual characters, avatars.

\section{INTRODUCTION}

This paper reports an experiment that examines the extent to which rules of proxemics are reproduced in an immersive virtual reality system when virtual characters enter the personal space of human participants. Earlier work in this field has investigated

This work was funded under the EU FET PRESENCCIA project contract Number 27731, with help from the Spanish Ministry of Science and Innovation. Joan Llobera's work was supported by the Torres Quevedo Program reference number PTQ06-1-0042

Authors' address: Joan Llobera, joan.llobera@ub.edu Facultat de Psicologia, Campus de Mundet - Edifici Teatre, Passeig de la Vall d'Hebron 171, 08035 Barcelona - Spain; www.event-lab.org, www.starlab.es

Permission to make digital/hard copy of part of this work for personal or classroom use is granted without fee provided that the copies are not made or distributed for profit or commercial advantage, the copyright notice, the title of the publication, and its date of appear, and notice is given that copying is by permission of the ACM, Inc. To copy otherwise, to republish, to post on servers, or to redistribute to lists, requires prior specific permission and/or a fee. 


\section{(c) 2001 ACM XXXX $\$ 5.00$}

proxemics in head-mounted display delivered virtual reality when participants walk towards a single virtual character or when a virtual character walks towards them [Bailenson et al. 2003; Bailenson et al. 2001]. The response measures were based on the actual movements of the participants (how close did they go to the virtual characters, how much did they move when the characters walked through them?). In [Wilcox et al. 2006] static stereo images of people and objects were used with response measures consisting of subjective reporting and skin conductance. In the experiment presented here we investigate mainly the skin conductance changes induced by varying the minimal distances that one or more virtual characters (either humanoid or cylindrical) approach to a participant who had been instructed to stand still. The virtual environment was delivered by a wide field of view head-tracked head-mounted display.

An ideal immersive virtual reality system supports perception through natural sensorimotor contingencies [Noë 2004], that is, those action-perception behaviors that humans employ in everyday life by using our bodies in order to perceive: we can bend down, stretch, reach out, move closer to or around an object or a whole environment, implicitly knowing the rules that relate perception to action. Current day head-tracked, wide field-of-view head-mounted displays or Cave-like systems [Cruz-Neira et al. 1993] support such sensorimotor contingencies to varying degrees. It has been noted in the literature that such systems can give rise to a feeling of being in the place depicted by the virtual environment displays, for example [Draper et al. 1998; Sanchez-Vives and Slater 2005; Sheridan 1992; Sheridan 1996; Slater 2009]. If people have this illusion and if what is depicted there is plausible then there is a tendency for people to respond realistically, across a range of parameters, to the virtual events and situations - even though they know for sure that they are experiencing an illusion. This has been demonstrated many times across a range of studies, for example [Bideau et al. 2003; Pertaub et al. 2002; Slater et al. 2006].

Here we have focused on human participant responses to virtual characters, in particular the extent to which people would conform to the implicit rules of distance between people as studied in the field of proxemics. This term, originally introduced by Hall [1973], describes four regions of interpersonal distance: public, social, personal and intimate, albeit modulated by cultural, gender and social status differences. It is postulated that the different distances of approach convey different social meanings and 
interpersonal relationships, forming a set of culturally bound implicit rules of distance. Therefore, when these rules are broken, a person might feel shocked and may attribute an erroneous intention to the person who has approached. It should be possible therefore to identify this shock with the measurement of behavioral, physiological and subjective reactions. Our main question is the extent to which proxemics behavior might be observed in virtual reality, giving credence to the view that people tend to respond realistically to virtual events and situations. From the existing literature it seems that this is likely to be the case - see for example, [Bailenson, Blascovich, Beall and Loomis 2003; Bailenson, Blascovich, Beall and Loomis 2001; Blascovich 2002; Blascovich et al. 2002; Friedman et al. 2007; Guye-Vuilleme et al. 1999; Wilcox, Allison, Elfassy and Grelik 2006].

Our experiment, discussed in detail in the following sections, has either 1 or 4 virtual characters repeatedly approaching a participant to varying distances (social, personal, and intimate). The characters approaching may be of two different types, either female virtual characters or cylinders of adult human size. Our response variables include physiological measures and subjective questionnaire reports. Our intention was to explore how these factors - proximity of approach, number of characters, number of approaches, and type of character - together influenced skin conductance response. We have presented a dynamic multi-character situation, with multiple repetitions - the latter serving also as a validation check on the skin conductance measures.

\section{PROXEMICS}

A relationship between proxemics and physiological responses was first reported by McBride [1965]. This work showed that varying the distances between two people influences skin conductance responses, and that one person having open or closed eyes, provokes significantly different responses in the skin conductance of the other. There is evidence that this also occurs in immersive virtual environments. Bailenson [Bailenson, Blascovich, Beall and Loomis 2001] showed that people spontaneously managed interpersonal differences differently when facing a graphical representation of a person compared to a cylinder, and that there were also significant effects related to the gaze direction of the character and other behavioral influences. 
It has also been shown that objects or people provoke different physiological responses consistent with proxemics theory when appearing at a fixed distance to the person looking at stereo images [Wilcox, Allison, Elfassy and Grelik 2006]. This was achieved with photorealistic representations of people displayed stereoscopically using a large screen $\left(2.35 \times 1.73 \mathrm{~m}^{2}\right)$ and it was shown to be consistent with the subjective reports of personal comfort. In [Wilcox, Allison, Elfassy and Grelik 2006], as well as in [Bailenson, Blascovich, Beall and Loomis 2001], a detailed overview of proxemics in non-mediated situations can be found. Using a different approach to the phenomenon, Beltran et al. [Beltran et al. 2006] showed that the spatial behavior of a crowd can be explained reasonably well with a bottom-up approach starting from proxemics rules. This demonstrated that the global behavior in a crowded space can be simulated using a local minimum discomfort level criterion, which is the difference between the desired ideal distance and the actual distance in a dyadic interaction. In the field of robotics Brooks and Arkin [Brooks and Arkin 2007] developed a behavioral model for a robot that took into account several affective aspects in relation to a nearby person in order to decide how the personal space was to be managed. In summary, previous work does suggest that basic rules of social interaction such as interpersonal distances in a virtual environment are maintained in the interaction with virtual characters.

The goal of our experiment was to assess whether people respond to virtual characters in similar ways to what would be predicted by proxemics, but in a dynamic situation where several virtual characters move but the human participant is stationary. Would the simultaneous approach of several virtual characters heighten the response compared to a single character? Would participants exhibit greater arousal in response to the approach of a human character compared to a non-human cylindrical one? There were, therefore, three main factors investigated: the distance of the approach, the type of the characters, and the number of characters who simultaneously approach. An important fourth factor, introduced as a validity check, was the number of times that virtual characters visited the participant. The results were assessed by using skin conductance measurements as well as by subjective questionnaires and reports. The experiment was approved by the ethical committee of the IDIBAPS at the Hospital Clinic of University of Barcelona. 


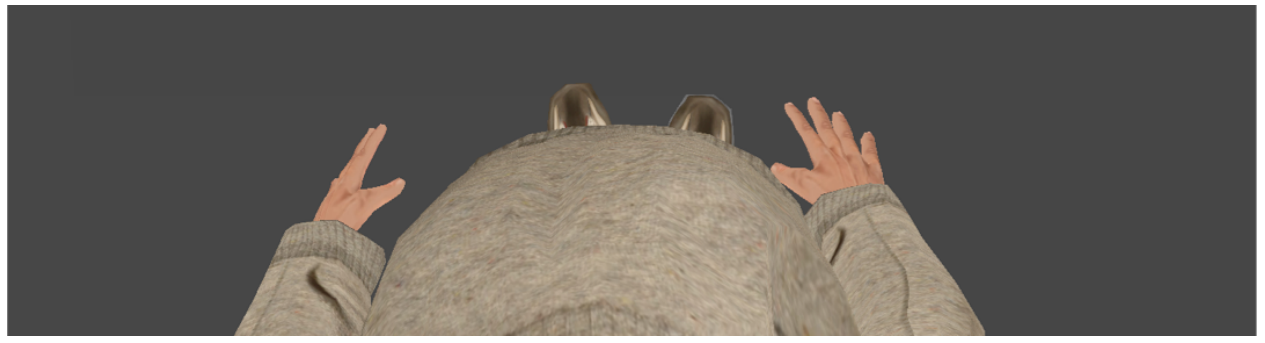

Figure 1: The virtual body of a participant as would be seen when he looked down at himself.

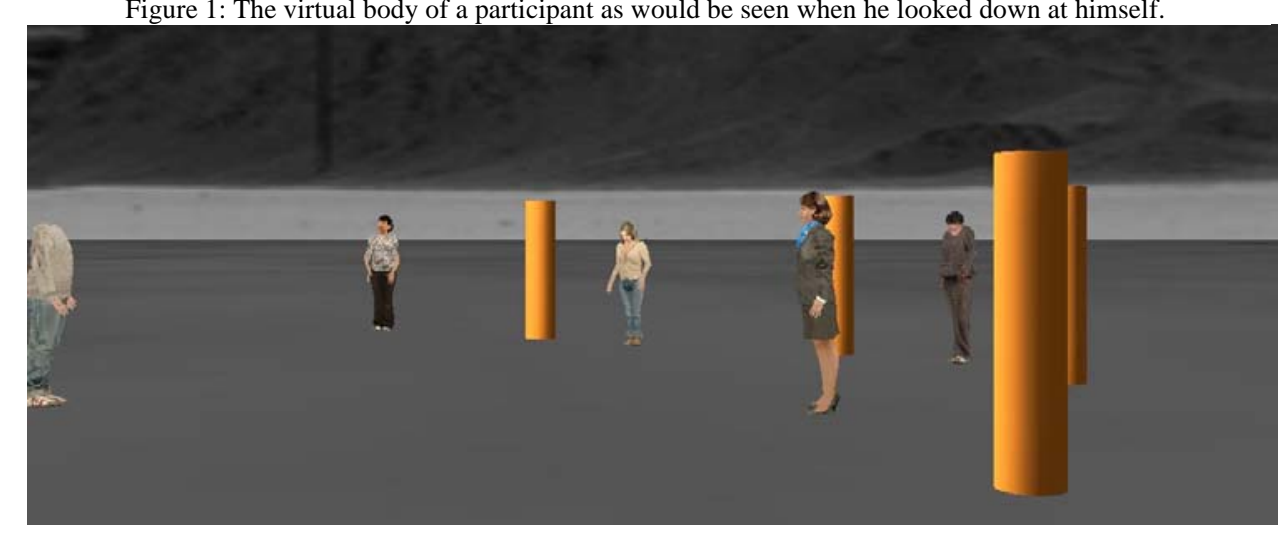

Figure 2: The characters and the cylinders involved in the experiment. At the left can be seen the virtual body that would be inhabited by the participant. 


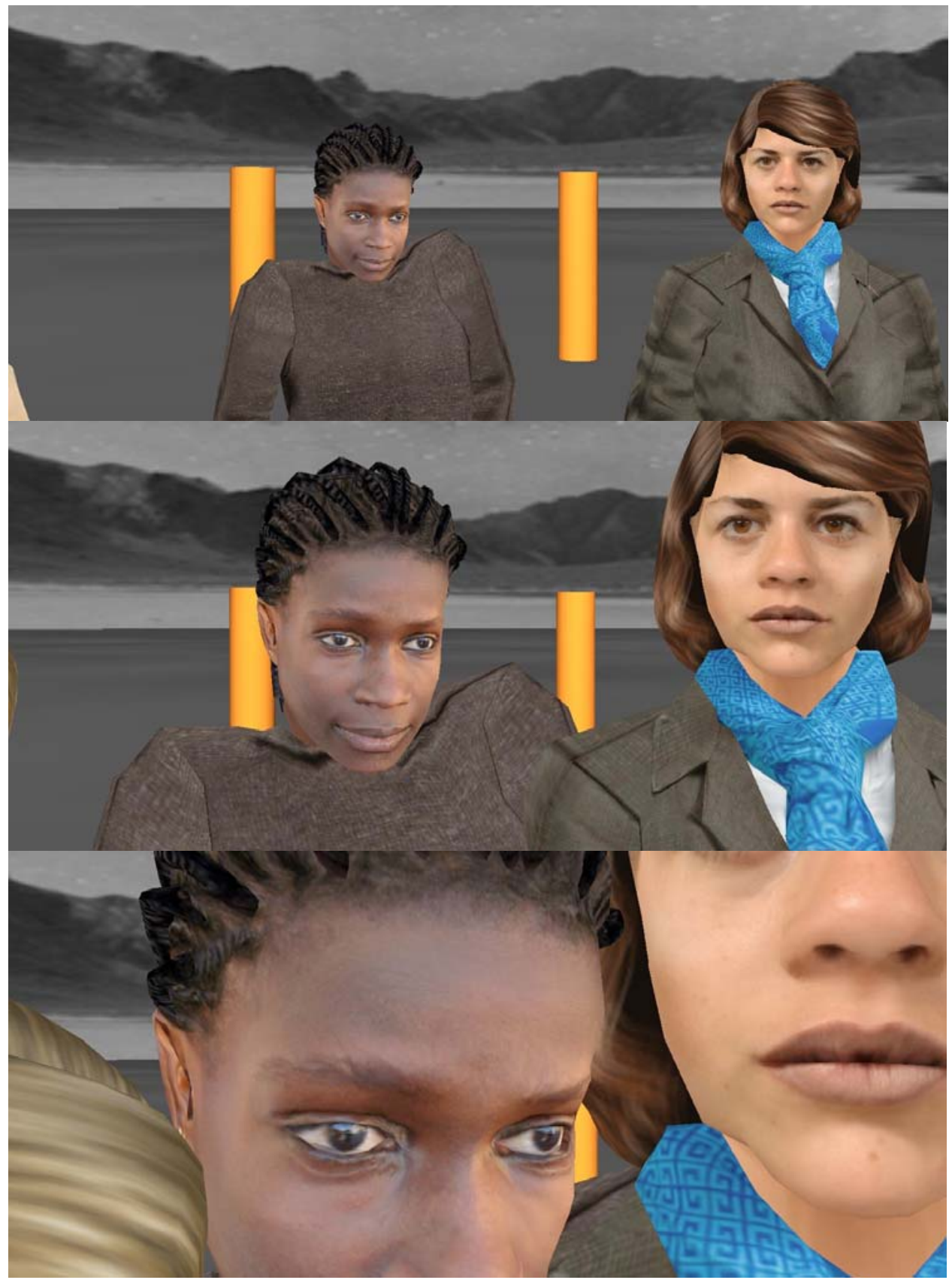

Figure 3: View of the characters terminating their walk at (a) 1.6, (b) 0.8 and (c) 0.4 meters respectively. The character postures are approximately the same at the end of each animation cycle. The orientation of the camera is the same throughout to facilitate the comparison. The participant would see all four characters through the wide field-of-view head-mounted display. The characters were not specifically programmed to look the participant in the eye, but this could occur sometimes by chance, for example the character to the right in the pictures. 


\section{MATERIALS AND METHODS}

\subsection{Participants}

Twelve participants were recruited by advertisement around the campus. Their age ranged between 22 and 39 years, and they were deliberately restricted to be males in order to remove one source of variation. The participants were paid 5 euros for their participation in the study.

\subsection{Materials}

A Fakespace Labs Wide5 head-mounted display was used which has field of view of $150^{\circ} \times 88^{\circ}$ with an estimated $1600 \times 1200$ resolution displayed at $60 \mathrm{~Hz}^{1}$, and also Sennheiser HD215 stereo headphones. The head-tracking system was an Intersense IS900, and the physiological recordings used a Mind Media Nexus 4 device, recording skin conductance at $128 \mathrm{~Hz}$.

The virtual environment was modeled by using 3D Studio Max to load motion captured data and export it appropriately to animate the virtual characters. The geometric, clothes and skin texture models of the virtual characters were acquired from the company AXYZ design $^{2}$. The Virtual Reality software system used was XVR [Carrozzino et al. 2005] and HALCA [Spanlang 2009] an extension of the Cal3D library for the character animation used to blend, loop and script the motions of the characters. The synchronization of all the devices and signals was achieved with a custom implementation that uses the VRPN protocol [Hudson et al. 2001].

\subsection{Procedure}

When a participant arrived for the experiment they were first given an information sheet describing its purpose and procedure. After reading this, and after the procedure had also been explained to them verbally, they were asked if they wished to continue and were then given a consent form to sign. Next the electrodes of the physiological recording device and the HMD were fitted. Participants wore headphones in order to further isolate

\footnotetext{
1 http://www.fakespacelabs.com/files/Download/Wide5\%20Data\%20Sheet.pdf

2 http://www.axyz-design.com/
} 
them from the laboratory and through which they would be able to hear wind noise and footsteps when the characters or the cylinders approached.

There was a baseline period of two minutes in order to give participants time to adjust to wearing and seeing through the HMD, and to become familiar with the scene, and also to record baseline physiological measures. During the baseline participants could see the characters standing far away, and were asked to describe what they were seeing in order to focus their attention on the surrounding elements. The characters were one of two types, female or cylinder, and during the baseline, the female ones made subtle movements to display life-like signs, but did not move otherwise. After the completion of the baseline the dynamic scenario started and participants were asked to stand in place and maintain silence. However, they were advised that they could leave the experiment at any time without giving reasons. The participants were head-tracked, and could move their head freely. After the end of the session they completed the post-experiment questionnaire, and a short interview was recorded.

\subsection{The scenario}

On entering the virtual reality via the HMD, participants would see that they had a virtual body, especially when they directly looked down at themselves (Figure 1). Since the HMD has a wide field of view participants could see their virtual body in peripheral vision much as in physical reality. The environment depicted an open field in which several humanoid characters and cylinders were standing at different distances from the participant, forming roughly a semi-circle of $4 \mathrm{~m}$ radius, as can be seen in Figures 2 and 3 . Then after the 2 minutes of baseline idling either 1 or a group of 4 characters (either all females or all cylinders) walked (or moved in the case of cylinders) towards the participant and (all) stopped at one of three possible distances. The cylinders followed the same paths as the humanoid characters that they replaced. They stood for 7 seconds at that distance and then went back to their initial position. We call each such approach a 'visit'.

\subsection{Experimental Design}

There were 4 factors with 12 subjects in a repeated-measures design. The factors were:

1. Number of characters: 1 or 4 characters approaching simultaneously. 
2. Distance: Since this factor is the most important one, 3 distances were selected: 1.6, 0.8 or 0.4 meters, which correspond approximately to the different proxemics categories (public, social or intimate).

3. Type: Each character could be either a cylinder or human female character.

4. Run number: The number of visits so far (between 1 and 12). This was used to track the habituation effect due to repeated visits.

Participants were assigned to combinations of the first three factors using a D-Optimal design [Dror and Steinberg 2006], and each participant always experienced 12 visits in total.

\subsection{Response Variables}

The main response variables were obtained from electrodermal activity (EDA). This measures changes in arousal through changes in skin conductance caused by sweat levels, reflecting changes in the sympathetic nervous system [Boucsein 1992]. An important derived measure of interest is the number of Skin Conductance Responses (SCR) which reflect transient sympathetic arousal, either spontaneous or in response to events, specifically the orienting response, that is responses to changes in the environment and events or surprises. Skin conductance responses (SCR) were defined to be local maxima that had amplitude of at least $0.1 \mu \mathrm{S}$ and in a period not exceeding $5 \mathrm{~s}$ from the start of the SCR to its maximal point. Such SCRs were identified in an offline program written in MATLAB. The number of SCRs was recorded for 10, 15 and 20 seconds after the arrival of the virtual characters at their destination in front of the participant. The number of SCRs in the baseline period was recorded to allow for differences between participants in the subsequent analysis.

A second measure derived from EDA was the maximum skin conductance level reached within $5 \mathrm{~s}$ after the characters had stopped walking and had arrived at their destination. As a baseline value we subtracted from this the participant's SCL at the moment that the characters were triggered by the computer program to start walking. Normally, one would take the maximum minus the level at the start of the event that might cause the rise (i.e., the moment of arrival of the characters at their destination in front of the participant). However, the situation here is more complex, since the start of the 'event' is fuzzy participants may become aroused at the moment they realize that the avatars are going to stop very close to themselves, in which case the SCL will already be rising by the time 
that they reach the destination. We call this measure the change in skin conductance level $\triangle$ SCL.

For an arousing event (for example, when the avatars approached to an intimate distance) we should find a greater number of SCRs compared to a non-arousing event, but, due to adaptation, we expect that with repetitions of the event the number of SCRs should decline. Also $\triangle \mathrm{SCL}$ should be greater for arousing than non-arousing events, and decline with repetitions.

Table I - Post experiment questionnaire (translated from Spanish). Each question was answered on a 0 to 10 scale where 0 meant 'not at all' and 10 meant 'very much so'.

\begin{tabular}{|l|l|}
\hline & overall experience of being in the virtual place \\
\hline 1 & The virtual world became the place where I felt I was located \\
\hline 2 & During the experiment I forgot about the real lab where this was happening \\
\hline 3 & During the experiment I felt that those events were really happening \\
\hline & responses to the approach of the characters \\
\hline 4 & $\begin{array}{l}\text { When people started approaching towards me, I hoped that they would stop before } \\
\text { arriving very near to me }\end{array}$ \\
\hline 5 & $\begin{array}{l}\text { When people stopped at a certain distance from me, it affected me less that when they } \\
\text { stopped very nearby }\end{array}$ \\
\hline 6 & It affected me more when there were more people approaching me \\
\hline 7 & When the people approached close to me, it had an effect on me \\
\hline & sense that the characters were real people \\
\hline 8 & During the experiment, I had the impression of being with real people \\
\hline 9 & Sometimes I forgot those people were not real \\
\hline 10 & Sometimes I had the sensation of feeling the body heat or smell of the people \\
\hline
\end{tabular}

\subsection{Questionnaire and Interview}

Immediately following their experience in the virtual reality scene the participants were given a questionnaire to complete, as shown in Table I. These were grouped into three types of question: the overall experience of being in the virtual place $(\mathrm{Q} 1,2,3)$, responses to the approach of the characters $(\mathrm{Q} 4,5,6,7)$, and the sense that the characters were real people $(\mathrm{Q} 8,9,10)$. A short informal interview was also conducted afterwards, and statements of the participants recorded. 


\subsection{Statistical Analysis}

Regression analysis was used to examine the relationship between the response variables and the four main factors described in Section 3.4. The first dependent variable considered is the number of SCRs per time period (10s, 15s, 20s). This is a discrete count variable, and under the null hypothesis that there is no effect of any of the independent variables, it should follow a Poisson distribution (the number of events occurring per unit time at random moments). The standard model here is therefore log-linear with a Poisson error structure which is a particular case of the class of Generalized Linear Models [McCullagh and Nelder 1989]. Normal regression analysis was used with the second dependent variable, $\triangle \mathrm{SCL}$, which, however, has the requirement that the residuals of the model fit follow a normal distribution, and this was found not to be the case. The usual approach in these circumstances is to find a monotone transformation of the response variable that resolves this problem. This was achieved with $-\exp (-\Delta \mathrm{SCL})$. In any case this transformation leads to qualitatively almost the same results as for the non transformed variable, as shown in the next section.

For all of the regressions we standardized the scores of the independent variables to have mean 0 and variance 1 . This does not result in any change in relationships nor in significance levels, but the utility is that when all of the independent variables are on the same scale the parameter estimates can be considered as effect sizes.

\section{RESULTS}

\subsection{Overview}

Figure 4 shows plots of the mean change in skin conductance ( $\triangle \mathrm{SCL})$ by the various factors. The effect of the baseline skin conductance has been eliminated by regression. Figure $4 \mathrm{~A}$ shows that the highest change in skin conductance is associated with the smallest distance of approach, and 4B that the skin conductance change is higher when 4 characters simultaneously approach than 1. 4C shows that there is an adaptation effect, that tis that the arousal declines sharply with repeated exposures. 4D suggests that there is no significant difference between the approach of female characters and cylinders. However, this analysis cannot take into account the simultaneous effects of all the variables, and for this we turn to multiple regression analyses in the next sections. 

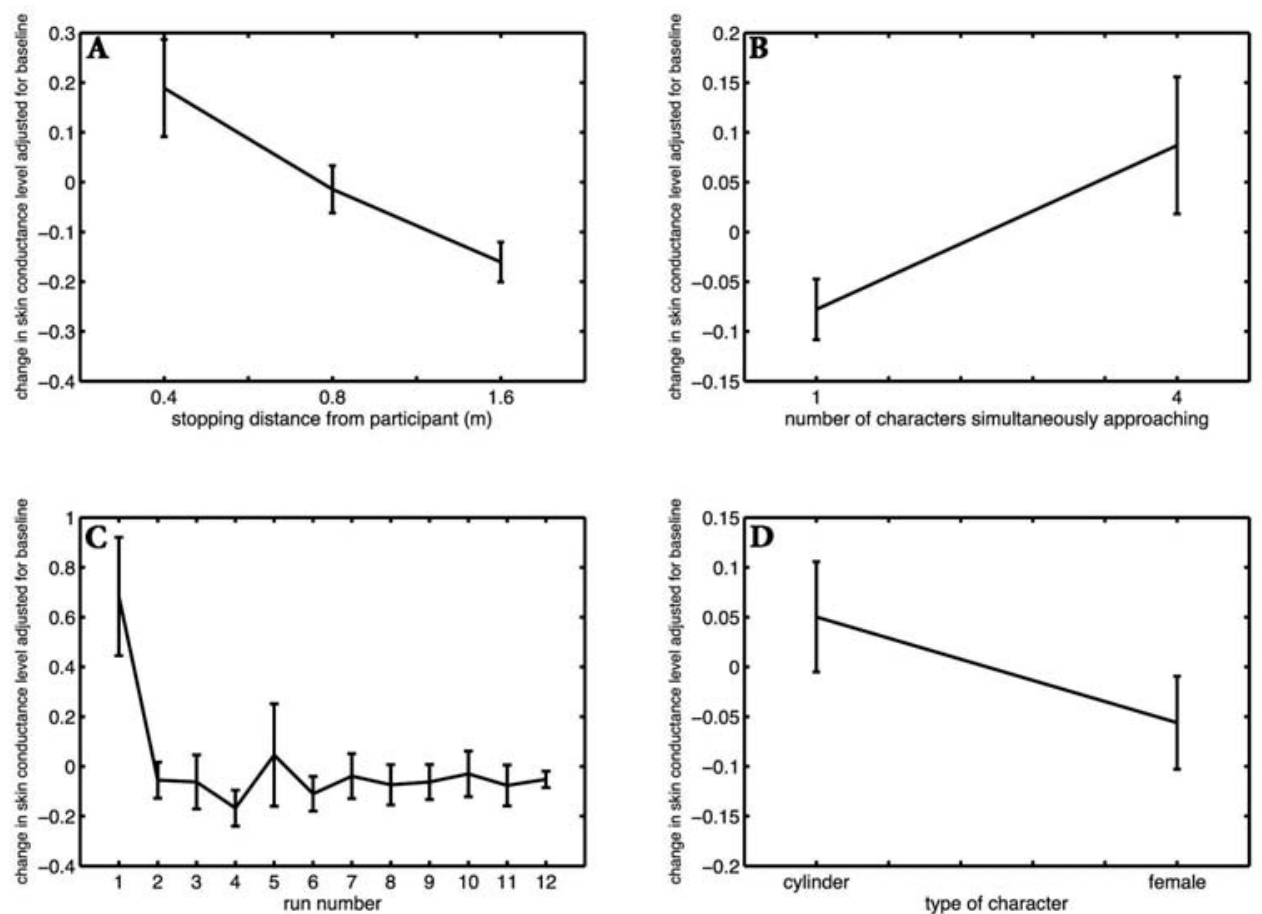

Figure 4 : Means and standard errors of the change in skin conductance (adjusted for the baseline) by

(A) stopping distance from the participant. (B) the number of characters approaching (1 or 4)

(C) the run number and (D) the type of character (cylinder or female).

\subsection{The number of SCRs}

The variance of the number of SCRs per 15s was more than double that of $10 \mathrm{~s}$, and the variance for $20 \mathrm{~s}$ was almost triple that for $10 \mathrm{~s}$. We present results for $20 \mathrm{~s}$ avoiding the problem of insufficient variation in the response variable - but actually the results for all these three possible response variables are very similar. We also included as an explanatory variable the number of SCRs during the baseline period, to account for $a$ priori differences between people.

Table II shows the result of the fit. The run number, that is the number of visits of the avatars so far, is negatively associated with the number of SCRs. This conforms to expectation since repetition of the stimulus leads to adaptation and therefore less arousal. Second, the closer the avatars were to the participant, the greater the arousal. This is consistent with what would be expected from proxemics theory. The number of characters simultaneously approaching has no effect. The baseline number of SCRs is clearly significant. The only curious result relates to the 'type'. When the number of SCRs per 10s is used this is not significant at all, and for $15 \mathrm{~s}$ the $\mathrm{P}$ value is 0.04 . Hence 
either the type is not significant or the humanoid characters are associated with less arousal than the cylinders - either way this is not what would be expected from proxemics theory. Amongst the relevant variables it is clear that distance has the greatest effect (its parameter estimate is 1.5 times that of run number).

Table II - Log Linear Regression for response variable: Number of SCRs per 20s after the arrival of the virtual characters to their stopping point in front of the participant.

The design matrix is standardized to mean 0 and variance 1 so that the parameter estimates are also comparative effect sizes.

Overall deviance of the fit is 102 on 138 d.f., $\mathrm{n}=144$.

\begin{tabular}{|l|c|l|}
\hline Term & $\begin{array}{c}\text { Parameter } \\
\text { Estimate }\end{array}$ & \\
\hline Constant & -0.066 & 0.417 \\
\hline Run number & -0.142 & 0.011 \\
\hline Distance & -0.213 & 0.001 \\
\hline Number of characters & -0.021 & 0.708 \\
\hline Type (Cylinders = 0, Virtual Females $=1)$ & -0.110 & 0.052 \\
\hline Baseline Number of SCRs & 0.956 & 0.000 \\
\hline
\end{tabular}

Table III -Linear Regression for response variable: ySCL $=-\exp (-\Delta S C L)$, where

$\triangle \mathrm{SCL}$ is the maximum skin conductance reading in $5 \mathrm{~s}$ following the arrival of the characters in front of the participant minus the skin conductance level at the moment that their walk started. The design matrix is standardized to mean 0 and variance 1 so that the parameter estimates are also comparative effect sizes.

For this model $\mathrm{R}^{2}=0.27$, overall $\mathrm{P}=0.000$, d.f. $=138, \mathrm{n}=144$.

\begin{tabular}{|l|c|c|}
\hline Term & $\begin{array}{c}\text { Parameter } \\
\text { Estimate }\end{array}$ & \\
\hline Constant & -0.858 & 0.000 \\
\hline Run number & -0.034 & 0.062 \\
\hline Distance & -0.064 & 0.001 \\
\hline Number of characters & 0.036 & 0.048 \\
\hline Gender (Cylinders, Virtual Females) & -0.024 & 0.178 \\
\hline Baseline Number of SCRs & 0.093 & 0.000 \\
\hline
\end{tabular}




\subsection{Change in Skin Conductance Level $(\Delta \mathrm{SCL})$}

A regression of $\triangle \mathrm{SCL}$ on the factors above leads to the same results for run number and distance, but in this case also the number of characters is positively associated with the response. The type is not significant at all. However, as stated earlier, the residuals of this fit do not follow a normal distribution. The transformation ySCL $=-\exp (-\Delta \mathrm{SCL})$ did result in normal residuals ( $\mathrm{P}=0.16$ with a Jarque-Bera test [Jarque and Bera 1980]), and the results are shown in Table III. The results are qualitatively the same as for Table I, but here there is some evidence to suggest that also the number of characters that simultaneously approach the participant is positively related to the change in skin conductance. The type is clearly not significant in this case.

\subsection{Questionnaire and Interview}

The questionnaire provides descriptive data with results given in Figure 5. On the whole participants had the illusion of being in the virtual place $(\mathrm{Q} 1,2)$, but not a strong illusion that the events were really happening (Q3). On the other hand their subjective impressions of how they responded when the characters approached them were quite consistent with the skin conductance results (Q4-7), even though consciously the characters were not considered as being real people (Q8-10).

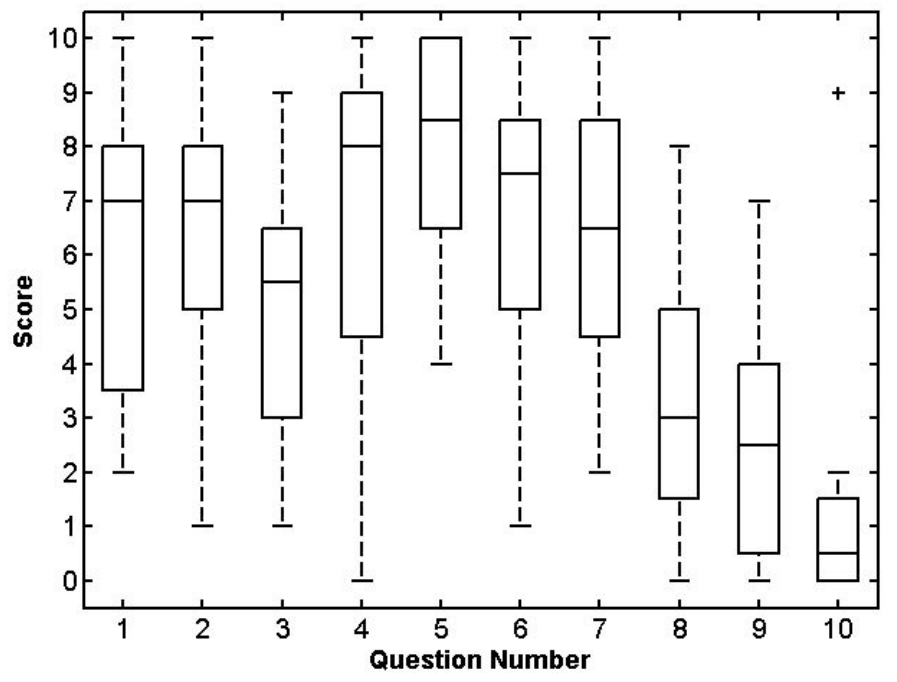

Figure 5 : Standard boxplots for the responses to the questionnaire. The medians are shown as the central horizontal bars, and the boxes are the interquartile ranges (IQR). The error bars (whiskers) represent either the extreme data points or extend to $1.5 \times \mathrm{IQR}$. There is one value outside the whisker for Q10 shown by $(+)$. 


\section{DISCUSSION}

Two different measures derived from the electrodermal activity recordings show that on average the shorter the distance of the characters from the participant, the greater the arousal. A supporting result of the validity of the skin conductance recordings was the finding that repetition reduced the level of arousal. There is also some evidence that the greater the number of characters who approached the greater the level of arousal. This is also backed up by the questionnaire results. These results are consistent with what would be expected from proxemics theory.

However, the unexpected result was that - with respect to EDA - participants tended to respond to cylinders in the same way as to the virtual people. As one participant wrote on the questionnaire: 'To see the columns coming close was more striking than the people, it gave me some fear, insecurity. When I saw people coming close to me I wanted to step back, but at the same time I knew it was not real, so I didn't do so (except maybe once). However, there was something very contradictory in my mind'. He said in the interview: 'In some way I knew the characters would stop, but I was not sure about the columns'. Other participants also said that the approach of the cylinders scared them.

These results are not the same as those found in [Bailenson, Blascovich, Beall and Loomis 2001] and [Wilcox, Allison, Elfassy and Grelik 2006]. Bailenson et al. found that people managed interpersonal distance differently if they had to approach a cylinder or a virtual character, but in their first experiment the virtual characters were static, and were approached by the participants. Wilcox et al. found that the physiological response to an object was less strong than to a face, but here both were also static. It is possible that dynamic, moving objects (in this case, cylinders) also evoke strong arousal responses when they approach humans. The arousal - although quantitatively the same as between humans and between these cylinders - is likely to represent qualitatively different mental processing. For example, a close approach by a virtual human may lead to discomfort because social norms are broken, whereas a close approach by a moving cylinder may lead to discomfort due to fear of collision. Moreover it should be noted that the cylinders had approximately the same dimensions as the people so it was not a question of size. Also, in physical reality, moving towards an adult human-sized cylinder is a very different experience than such a cylinder moving towards you. 
Another possible interpretation is that proxemics rules applied to cylinders because of the nature of the movement. In the experiment, the cylinders followed the same trajectory as the female characters they replaced. This gave them a more saccade-like path that could make them intuitively be understood as social agents.

Studies of proxemics in virtual reality have usually focused on static environments in the sense that it is the participants move, not the virtual characters. Our experiment shows that it is likely that environments with dynamic virtual characters are more complex to understand. There has to be a clear separation between the social aspects (this person is too close to me) and the physical aspects (this potentially dangerous object is too close me). It is clear that in these environments simple interpretations of physiological responses such as skin conductance are not sufficient to determine social cause. However, in our case the subjective response results are consistent with the results found from the physiological measures.

Although the results have good statistical significance, further work is needed to understand more clearly why the response to cylinders differs from the accounts given in literature, as well as analyzing the combined effect of other factors such as gaze, gender or even cultural context, which are known to affect the reaction provoked by interpersonal distances.

\section{REFERENCES}

BAILENSON, J., BLASCOVICH, J., BEALL, A. and LOOMIS, J. 2003. Interpersonal Distance in Immersive Virtual Environments. Personality and Social Psychology Bulletin 29, 819-833.

BAILENSON, J.N., BLASCOVICH, J., BEALL, A.C. and LOOMIS, J.M. 2001. Equilibrium theory revisited: Mutual gaze and personal space in virtual environments. Presence: Teleoperators \& Virtual Environments 10, 583-598.

BELTRAN, F.S., SALAS, L. and QUERA, V. 2006. Spatial Behavior in Groups: an Agent-Based Approach. Jasss-the Journal of Artificial Societies and Social Simulation 9, - .

BIDEAU, B., KULPA, R., MENARDAIS, S., FRADET, L., MULTON, F., DELAMARCHE, P. and ARNALDI, B. 2003. Real handball goalkeeper vs. virtual handball thrower. Presence-Teleoperators and Virtual Environments 12, 411-421.

BLASCOVICH, J. 2002. Social influence within immersive virtual environments. Springer CSCW Series, 127-145. 
BLASCOVICH, J., LOOMIS, J., BEALL, A., SWINTH, K., HOYT, C. and BAILENSON, J. 2002. Immersive Virtual Environment Technology as a Methodological Tool for Social Psychology. Psychological Inquiry 13, 103-124.

BOUCSEIN, W. 1992. Electrodermal Activity. Plenum Press, New York.

BROOKS, A.G. and ARKIN, R.C. 2007. Behavioral overlays for non-verbal communication expression on a humanoid robot. Autonomous Robots 22, 55-74.

CARROZZINO, M., TECCHIA, F., BACINELLI, S., CAPPELLETTI, C. and BERGAMASCO, M. 2005. Lowering the development time of multimodal interactive application: the real-life experience of the XVR project. In Proceedings of the 2005 ACM SIGCHI International Conference on Advances in computer entertainment technology ACM Press New York, NY, USA, 270-273.

CRUZ-NEIRA, C., SANDIN, D.J. and DEFANTI, T.A. 1993. Surround-screen projection-based virtual reality: the design and implementation of the CAVE. Proceedings of the 20th annual conference on Computer graphics and interactive techniques, 135-142.

DRAPER, J.V., KABER, D.B. and USHER, J.M. 1998. Telepresence. Human Factors 40, 354-375.

DROR, H.A. and STEINBERG, D.M. 2006. Robust experimental design for multivariate generalized linear models. Technometrics 48, 520-529.

FRIEDMAN, D., STEED, A. and SLATER, M. 2007. Spatial Social Behavior in Second Life. LECTURE NOTES IN COMPUTER SCIENCE 4722, 252.

GUYE-VUILlEME, A., CAPIN, T.K., PANDZIC, S., THALMANN, N.M. and THALMANN, D. 1999. Nonverbal communication interface for collaborative virtual environments. Virtual Reality 4, 49-59.

HALL, E.T. 1973. The hidden dimension. Leonardo, 94-94.

HUDSON, T.C., SEEGER, A., WEBER, H., JULIANO, J. and HELSER, A.T. 2001. VRPN: a device-independent, network-transparent VR peripheral system ACM New York, NY, USA, 55-61.

JARQUE, C.M. and BERA, A.K. 1980. Efficient tests for normality, homoscedasticity and serial independence of regression residuals. Economics Letters 6, 255-259.

MCBRIDE, G., KING, M.G. and JAMES, J.W. 1965. Social proximity effects on galvanic skin responses in adult humans. The Journal of psychology 61, 153.

MCCULLAGH, P. and NELDER, J.A. 1989. Generalized linear models. Chapman and Hall, London ; New York.

NOË, A. 2004. Action In Perception. Bradford Book. 
PERTAUB, D.P., SLATER, M. and BARKER, C. 2002. An experiment on public speaking anxiety in response to three different types of virtual audience. PresenceTeleoperators and Virtual Environments 11, 68-78.

SANCHEZ-VIVES, M.V. and SLATER, M. 2005. From Presence to Consciousness through Virtual Reality. Nature Reviews Neuroscience 6, 332-339.

SHERIDAN, T.B. 1992. Musings on Telepresence and Virtual Presence. Presence: Teleoperators and Virtual Environments 1, 120-126.

SHERIDAN, T.B. 1996. Further musings on the psychophysics of presence. Presence: Teleoperators and Virtual Environments 5, 241-246.

SLATER, M. 2009. Place Illusion and Plausibility Can Lead to Realistic Behaviour in Immersive Virtual Environments. Philos Trans $R$ Soc Lond in press.

SLATER, M., ANTLEY, A., DAVISON, A., SWAPP, D., GUGER, C., BARKER, C., PISTRANG, N. and SANCHEZ-VIVES, M.V. 2006. A virtual reprise of the Stanley Milgram obedience experiments. PLoS ONE 1, e39. doi:10.1371/journal.pone.0000039.

SPANLANG, B. 2009. HALCA: A Hardware accelerated library for character animation EVENT Lab, Facultat de Psicologia, University of Barcelona, Barcelona.

WILCOX, L.M., ALLISON, R.S., ELFASSY, S. and GRELIK, C. 2006. Personal space in virtual reality. ACM Transactions on Applied Perception (TAP) 3, 412-428. 